\title{
Finite-time thermodynamics of fluctuations in microscopic heat engines
}

\author{
Gentaro Watanabe $\oplus^{1,2}$ and Yuki Minami $\odot^{1}$ \\ ${ }^{1}$ Department of Physics and Zhejiang Institute of Modern Physics, Zhejiang University, Hangzhou, Zhejiang 310027, China \\ ${ }^{2}$ Zhejiang Province Key Laboratory of Quantum Technology and Device, Zhejiang University, Hangzhou, Zhejiang 310027, China
}

(Received 19 August 2021; revised 15 November 2021; accepted 6 January 2022; published 31 January 2022)

\begin{abstract}
Fluctuations of thermodynamic quantities become non-negligible and play an important role when the system size is small. We develop finite-time thermodynamics of fluctuations in microscopic heat engines whose environmental temperature and mechanical parameter are driven periodically in time. Within the slow-driving regime, this formalism universally holds in a coarse-grained timescale whose resolution is much longer than the correlation time of the fluctuations, and is shown to be consistent with the relation analogous to the fluctuation-dissipation relation. Employing a geometric argument, a scenario to simultaneously minimize both the average and fluctuation of the dissipation in the Carnot cycle is identified. For this simultaneous optimization, the existence of a zero eigenvalue of the singular metric for the scale invariant equilibrium state is found to be essential. Furthermore, we demonstrate that our optimized protocol can improve the dissipation and its fluctuation over the current experiment.
\end{abstract}

DOI: 10.1103/PhysRevResearch.4.L012008

Introduction. Technological development has enabled us to fabricate heat engines using small systems [1-9]. To understand the performance of such small engines, theory beyond the conventional thermodynamics for macroscopic systems, thermodynamics of small systems, is called for. Recent synergy between technology and the remarkable success of stochastic thermodynamics [10-16] in understanding thermodynamic properties of small systems [17] including micromachines using active matter [18-20] and biological systems [21] has led to a surge of activity on the study of microscopic heat engines [22-28], where effects of thermal fluctuation can play an important role [29-59].

For reversible heat engines under quasistatic operations, thermodynamics provides the celebrated Carnot bound on their efficiency, and some universal relations are also known even for fluctuations [38]. However, in the practical situations, heat engines are operated within a finite period of cycle. There, losses due to dissipation is inevitable, and how to reduce such dissipative loss is a crucial issue. Furthermore, since the amount of the dissipative loss in microscopic heat engines significantly varies for each trial (e.g., the fluctuation of the efficiency is of order unity in the experiment of Ref. [5]), how to suppress the fluctuation of the dissipation is an equally important agenda, which stands out as an open problem. Here, we formulate finite-time thermodynamics of fluctuations in microscopic heat engines which is applicable to the slow-driving regime and coarse-grained timescale. This formalism provides us a geometric description for fluctuation

Published by the American Physical Society under the terms of the Creative Commons Attribution 4.0 International license. Further distribution of this work must maintain attribution to the author(s) and the published article's title, journal citation, and DOI. of the dissipation under finite-time operations. Geometric descriptions [60-66] of the mean value of the dissipative loss are known for both the macroscopic [67-69] and microscopic systems $[27,70-73]$. It is noted that such a geometric description is also possible for its fluctuation. We apply our framework to the current experiment and provide an optimum scheme to minimize both the mean and the fluctuation of the dissipation simultaneously unlike trade-off optimization (see also [74] for trade-off optimization between work fluctuation and efficiency and [75-78] for recent works on optimization of the mean value). Interestingly, it is found that the scale invariance of the equilibrium state is essential for such a simultaneous optimization, and this optimization is possible only for cycles solely consisting of isothermal and isentropic strokes as the Carnot cycle. Since the dissipation is closely related to the efficiency, which is also fluctuating in microscopic heat engines, the reduction of its mean and fluctuation leads to an enhancement and stabilization of the efficiency.

Setup. Consider a classical microscopic heat engine whose working substance is always in contact with an environment whose temperature $T$ is controllable. This is a standard setting for the experiments of the classical microscopic heat engines [3,5,7,9]. The Hamiltonian $H_{\lambda_{w}}$ of the working substance has an external mechanical parameter $\lambda_{w}$ (or $V$ ). Therefore, the system has two control parameters, $\lambda_{w}$ and $\lambda_{u} \equiv T$, and the engine protocol is specified by a closed path $\mathcal{C}$ in the parameter space spanned by the vector $\lambda_{\mu} \equiv\left(\lambda_{w}, \lambda_{u}\right)$. The parameters are varied cyclically to drive the state (more precisely, the phase space distribution function $p$ ) of the working substance to be periodic in time with the period $\tau$ of the cycle.

The generalized forces $\left(X_{w}, X_{u}\right)$ conjugate to the parameters $\left(\lambda_{w}, \lambda_{u}\right)$ are $\left(X_{w}, X_{u}\right) \equiv(P, S)=\left(-\partial H_{\lambda_{w}} / \partial \lambda_{w},-\ln p\right)$, where $P \equiv-\partial H_{\lambda_{w}} / \partial \lambda_{w}$ is the generalized pressure and $S \equiv$ $-\ln p$ is the stochastic entropy. Note that $X_{w}$ and $X_{u}$ are random variables as functions of the phase space point $\Gamma$. 
The loss of the energy due to driving with nonzero speed is quantified by the dissipated availability $A$ introduced by Salamon and Berry [67]: $A \equiv U-W$ with $W$ being the work output by the engine and $U$ being the effective energy input from the environment (not to be confused with the internal energy) defined respectively as

$$
\begin{aligned}
W & \equiv \oint_{\mathcal{C}} P d V=\int_{0}^{\tau} d t\left(-\frac{\partial H_{\lambda_{w}}}{\partial \lambda_{w}}\right) \dot{\lambda}_{w}=\int_{0}^{\tau} d t X_{w} \dot{\lambda}_{w}, \\
U & \equiv \oint_{\mathcal{C}} T d S=\int_{0}^{\tau} d t T \frac{d}{d t}(-\ln p)=\int_{0}^{\tau} d t \lambda_{u} \dot{X}_{u},
\end{aligned}
$$

where the dot denotes the time derivative. Here, $A, W$, and $U$ are random variables. Note that $T$ in $U$ is the temperature of the environment instead of that of the working substance, and $U$ is different from heat input in general, but agrees with the latter in the quasistatic limit.

In the following, we discuss the fluctuation of random variables. Fluctuation $\Delta Y$ of a random variable $Y$ is given by $\Delta Y \equiv Y-\langle Y\rangle$, where $\langle\ldots\rangle$ means an ensemble average.

Since the working substance is always in contact with the thermal environment, correlations of the physical quantities decay exponentially in time provided the driving is slow enough. In the coarse-grained timescale whose resolution is much longer compared to the decay time, such an exponential decay function can be well approximated by a delta function multiplied by its decay time constant (or the correlation time). Therefore, in the slow-driving regime and coarse-grained timescale, the two-time correlation function of the fluctuations could universally be written in the form of

$$
\left\langle\Delta X_{\mu}(t) \Delta X_{v}\left(t^{\prime}\right)\right\rangle=2\left\langle\Delta X_{\mu}(t) \Delta X_{v}(t)\right\rangle \tau_{\mu v}(t) \delta\left(t-t^{\prime}\right),
$$

where $\tau_{\mu \nu}(t)\left[=\tau_{\nu \mu}(t)\right]$ is the correlation time between $\Delta X_{\mu}$ and $\Delta X_{v}$ at time $t$. In the following, we will formulate finite-time thermodynamics of fluctuations of the dissipated availability based on Eq. (3). The ansatz (3) is the minimal prescription to introduce the time scale to thermodynamics through $\tau_{\mu \nu}$ which depends on microscopic details of the system.

First, we consider the variance $\left\langle\Delta A^{2}\right\rangle$ of $A$ for a cycle given by $\left\langle\Delta A^{2}\right\rangle=\left\langle\Delta U^{2}\right\rangle+\left\langle\Delta W^{2}\right\rangle-2\langle\Delta U \Delta W\rangle$. Regarding the variance of $W$, using Eq. (3) and the condition of the closed cycle such that the phase space distribution function at $t=0$ and $\tau$ are the same, $p(0)=p(\tau)$, we obtain

$$
\begin{aligned}
\left\langle\Delta W^{2}\right\rangle= & \int_{0}^{\tau} d t \int_{0}^{\tau} d t^{\prime}\left[\left\langle X_{w}(t) X_{w}\left(t^{\prime}\right)\right\rangle-\left\langle X_{w}(t)\right\rangle\left\langle X_{w}\left(t^{\prime}\right)\right\rangle\right] \\
& \times \dot{\lambda}_{w}(t) \dot{\lambda}_{w}\left(t^{\prime}\right) \\
= & 2 \int_{0}^{\tau} d t\left\langle\Delta X_{w}^{2}(t)\right\rangle \tau_{w w}(t) \dot{\lambda}_{w}^{2}(t)
\end{aligned}
$$

By integrating by parts, $U$ given by Eq. (2) can be rewritten as $U=-\int_{0}^{\tau} X_{u}(t) \dot{\lambda}_{u}(t) d t+\left[X_{u}(\tau)-X_{u}(0)\right] \lambda_{u}(0)$ since $\lambda_{u}(\tau)=\lambda_{u}(0)$ but $X_{u}(\tau) \neq X_{u}(0)$ in general. Thus, using Eq. (3) and the closed cycle condition as in the way to get Eq. (4), we obtain

$$
\left\langle\Delta U^{2}\right\rangle=2 \int_{0}^{\tau} d t\left\langle\Delta X_{u}^{2}(t)\right\rangle \tau_{u u}(t) \dot{\lambda}_{u}^{2}(t)+2\left\langle\Delta X_{u}^{2}(0)\right\rangle \lambda_{u}^{2}(0),
$$

$$
\langle\Delta W \Delta U\rangle=-2 \int_{0}^{\tau} d t\left\langle\Delta X_{w}(t) \Delta X_{u}(t)\right\rangle \tau_{w u}(t) \dot{\lambda}_{w}(t) \dot{\lambda}_{u}(t) .
$$

From Eqs. (4)-(6), we finally obtain

$$
\left\langle\Delta A^{2}\right\rangle=\int_{0}^{\tau} d t g_{\mu \nu}^{(2)}(t) \dot{\lambda}_{\mu}(t) \dot{\lambda}_{\nu}(t)+2\left\langle\Delta X_{u}^{2}(0)\right\rangle \lambda_{u}^{2}(0)
$$

with

$$
g_{\mu \nu}^{(2)}(t) \equiv 2 \tau_{\mu \nu}(t)\left\langle\Delta X_{\mu}(t) \Delta X_{\nu}(t)\right\rangle \equiv 2 \tau_{\mu \nu}(t) \sigma_{\mu \nu}(t)
$$

for each $(\mu, v)$ element, where $\sigma_{\mu \nu} \equiv\left\langle\Delta X_{\mu}(t) \Delta X_{v}(t)\right\rangle$ is the covariance matrix element between $X_{\mu}$ and $X_{v}$. Summation should be taken over $(w, u)$ components for the repeated $\mu$ and $v$ in Eq. (7) and hereafter. For a continuous operation taking over consecutive $N$ cycles, an average $\overline{\left\langle\Delta A^{2}\right\rangle}$ of the fluctuation of $A$ per cycle in the limit of $N \rightarrow \infty$ is given by

$$
\begin{aligned}
\overline{\left\langle\Delta A^{2}\right\rangle} & =\lim _{N \rightarrow \infty} \frac{1}{N} \int_{0}^{N \tau} d t g_{\mu \nu}^{(2)}(t) \dot{\lambda}_{\mu}(t) \dot{\lambda}_{\nu}(t) \\
& =\int_{0}^{\tau} d t g_{\mu \nu}^{(2)}(t) \dot{\lambda}_{\mu}(t) \dot{\lambda}_{\nu}(t),
\end{aligned}
$$

since the second term of $\left\langle\Delta A^{2}\right\rangle$ in Eq. (7) comes solely from the end points and their contribution vanishes by taking the average over an infinite number of cycles.

Since the covariance matrix $\sigma_{\mu \nu}$ is positive definite, $g_{\mu \nu}^{(2)}$ is also positive definite when $\tau_{w w} \tau_{u u}=\tau_{w u}^{2}$. Therefore, as long as $\tau_{w w} \tau_{u u}=\tau_{w u}^{2}$ is satisfied, $g_{\mu \nu}^{(2)}$ can be regarded as a metric tensor. Note that the resulting expression of the variance $\overline{\left\langle\Delta A^{2}\right\rangle}$ given by Eq. (9) is in the similar form as the average $\langle A\rangle=\int_{0}^{\tau} d t g_{\mu \nu}^{(1)}(t) \dot{\lambda}_{\mu}(t) \dot{\lambda}_{v}(t)$ derived in Ref. [27], but with a different metric tensor $g_{\mu \nu}^{(2)}$. From the Cauchy-Schwarz inequality, $\left[\int d t f(t) g(t)\right]^{2} \leqslant\left[\int d t f^{2}(t)\right]\left[\int d t g^{2}(t)\right]$ with $f(t)=\left(g_{\mu \nu}^{(2)} \dot{\lambda}_{\mu} \dot{\lambda}_{\nu}\right)^{1 / 2}$ and $g(t)=1$, we get

$$
\overline{\left\langle\Delta A^{2}\right\rangle} \geqslant \frac{\left(\mathcal{L}^{(2)}\right)^{2}}{\tau}
$$

with the thermodynamic length $\mathcal{L}^{(2)}$ for the variance of $A$ :

$$
\mathcal{L}^{(2)} \equiv \int_{0}^{\tau} d t \sqrt{g_{\mu \nu}^{(2)}(t) \dot{\lambda}_{\mu}(t) \dot{\lambda}_{\nu}(t)}=\oint \sqrt{g_{\mu \nu}^{(2)} d \lambda_{\mu} d \lambda_{\nu}} .
$$

The equality in Eq. (10) holds if and only if $g_{\mu \nu}^{(2)}(t) \dot{\lambda}_{\mu}(t) \dot{\lambda}_{v}(t)$ is constant or identically equal to zero.

Relation between $g_{\mu \nu}^{(1)}$ and $g_{\mu \nu}^{(2)}$. Next, we discuss the relation between $g_{\mu \nu}^{(1)}$ and $g_{\mu \nu}^{(2)}$. Here, we assume that the phase space distribution function $p$ follows the Fokker-Planck equation (or more generally, the Kramers-Moyal equation):

$$
\frac{\partial p(\Gamma, t)}{\partial t}=L(\Gamma, t) p(\Gamma, t)
$$

with $L(\Gamma, t)$ being the derivative operator (so-called the Fokker-Planck operator or the Kramers-Moyal operator), so that the equilibrium distribution $p^{\text {eq }}$ satisfies the detailed balance condition:

$$
L(\Gamma, t) p^{\mathrm{eq}}(\Gamma) \ldots=p^{\mathrm{eq}}(\Gamma) L^{\dagger}(\sigma \Gamma, t) \ldots,
$$

where $\dagger$ denotes the adjoint and $\sigma(=+1$ or -1$)$ is the symmetry factor of the phase space variable under the time 
reversal operation. For sufficiently slow driving such that its time scale $\lambda_{\mu} / \dot{\lambda}_{\mu}$ is much larger than the relaxation time of the working substance, we write

$$
p(\Gamma, t)=[1+\xi(\Gamma, t)] p^{\mathrm{eq}}(\Gamma ; t),
$$

where $p^{\mathrm{eq}}(\Gamma ; t) \equiv \exp \left[-\beta(t) H_{\lambda_{w}}(\Gamma ; t)\right] / Z_{t}$ is the equilibrium state for the instantaneous parameter values $\left(\lambda_{w}(t), \lambda_{u}(t)\right)$ at $t, \beta(t) \equiv 1 / T(t)$ is the instantaneous inverse temperature, $Z_{t} \equiv \int d \Gamma \exp \left[-\beta(t) H_{\lambda_{w}}(\Gamma ; t)\right]$ is the partition function, and $\xi \ll 1$ describes the deviation from $p^{\mathrm{eq}}$. Within the linear approximation with respect to the small quantities $\dot{\lambda}_{\mu}$ and $\xi$, we obtain (see [79] for details)

$\xi(\Gamma, t) \simeq-\beta(t) \int_{0}^{\infty} d s e^{L^{\dagger}(\sigma \Gamma, t) s}\left[X_{\mu}(t)-\left\langle X_{\mu}(t)\right\rangle_{t, \mathrm{eq}}\right] \dot{\lambda}_{\mu}(t)$

with $\langle\ldots\rangle_{t, \text { eq }} \equiv \int d \Gamma p^{\mathrm{eq}}(\Gamma ; t) \ldots$ being the average for the instantaneous equilibrium state at $t$. Within the linear response regime, the metric tensor $g_{\mu \nu}^{(1)}$ for $\langle A\rangle$ can be written as $g_{\mu \nu}^{(1)}(t) \equiv-2^{-1}\left[R_{\mu \nu}(t)+R_{\nu \mu}(t)\right]$ with the response coefficients $R_{\mu \nu}$ defined by $\left\langle X_{\mu}(t)\right\rangle=\int d \Gamma X_{\mu}(t)(1+\xi) p^{\mathrm{eq}} \equiv$ $\left\langle X_{\mu}(t)\right\rangle_{t \text {, eq }}+R_{\mu \nu}(t) \dot{\lambda}_{\nu}(t)$ [27]. Using Eq. (15), it can be shown that the ansatz (3) leads to the relation between the two metric tensors $g_{\mu \nu}^{(1)}$ and $g_{\mu \nu}^{(2)}$ as

$$
g_{\mu \nu}^{(2)}(t)=2 T(t) g_{\mu \nu}^{(1)}(t)
$$

which is analogous to the fluctuation-dissipation relation.

Application to the Brownian Carnot cycle. An overdamped Brownian particle in a harmonic oscillator trapping potential is a typical setup commonly employed in experiments of microscopic heat engines $[3,5,7,9,13]$. Taking this setup as an example, we shall apply our framework to evaluate the fluctuation of the performance of the microscopic heat engine. For simplicity, we consider a one-dimensional case, and thus the phase space point $\Gamma$ of the overdamped system can be specified solely by the position of the Brownian particle $q$ : $\Gamma=\{q\}$. Suppose, with the mechanical control parameter $\lambda_{w}$, the external one-dimensional harmonic oscillator trap $V_{\lambda_{w}}(q)$ is given by

$$
V_{\lambda_{w}}(q)=\frac{\lambda_{w}}{2} q^{2}
$$

This system can be described by the time-dependent OrnsteinUhlenbeck process whose Fokker-Planck equation is $[80,82]$

$$
\frac{\partial}{\partial t} p(q, t)=\frac{\lambda_{w}(t)}{\gamma} \frac{\partial}{\partial q}[q p(q, t)]+\frac{D(t)}{\gamma} \frac{\partial^{2}}{\partial q^{2}} p(q, t),
$$

where $D$ is the diffusion constant and $\gamma$ is the friction coefficient.

Now, we consider a cycle whose driving speed $\dot{\lambda}_{\mu}$ is finite, but sufficiently slow (i.e., the time scale $\lambda_{\mu} / \dot{\lambda}_{\mu}$ is much larger than the relaxation time) so that the working substance is always close to the instantaneous equilibrium state and

$$
D(t)=\beta^{-1}(t) \text {. }
$$

Thus, to obtain $\langle A\rangle$ and $\overline{\left\langle\Delta A^{2}\right\rangle}$ up to the linear order of small quantities, $g_{\mu \nu}^{(i)}$ in their expressions can be evaluated for the instantaneous equilibrium state since it is already multiplied by a factor of the linear order of small quantities: $\int_{0}^{\tau} d t \dot{\lambda}_{\mu} \dot{\lambda}_{v}$.
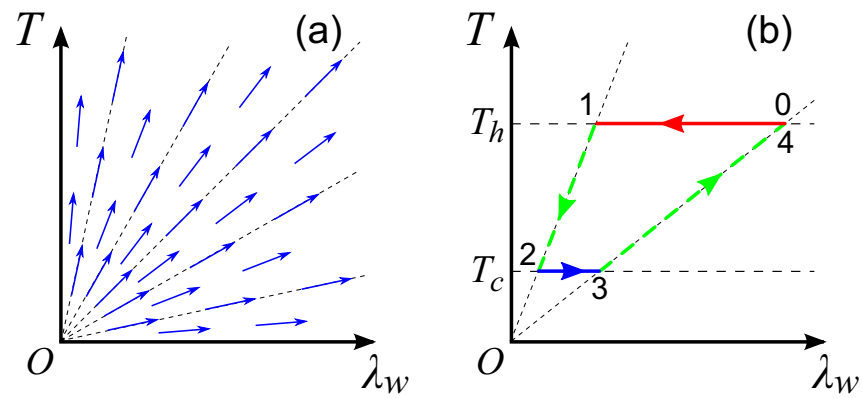

FIG. 1. (a) Eigenvector field of $g_{\mu \nu}^{(2)}$ corresponding to the zero eigenvalue shown in the $T-\lambda_{w}$ plane. The dotted straight lines are a guide for the eye showing the directions of the zero eigenvalue of $g_{\mu \nu}^{(2)}$ for different values of $T / \lambda_{w}$. (b) Brownian Carnot cycle, which consists of two isothermal strokes at $T_{h}(0 \rightarrow 1)$ and $T_{c}(2 \rightarrow 3)$, and two isentropic strokes $(1 \rightarrow 2$ and $3 \rightarrow 4)$.

Since the Hamiltonian of the overdamped system is given solely by the potential energy, i.e., $H_{\lambda_{w}}=V_{\lambda_{w}}$, the instantaneous equilibrium state is $p^{\mathrm{eq}}=\exp \left[-\beta \lambda_{w} q^{2} / 2\right] / Z_{t}$ with $Z_{t}=\sqrt{2 \pi T / \lambda_{w}}$. For the equilibrium state, each element of the covariance matrix $\sigma_{\mu v}=\left\langle\Delta X_{\mu} \Delta X_{\nu}\right\rangle_{t \text {, eq }}$ reads $\sigma_{w w}=$ $\left(T / \lambda_{w}\right)^{2} / 2, \sigma_{w u}=\sigma_{u w}=-T / 2 \lambda_{w}$, and $\sigma_{u u}=1 / 2$.

Next, we consider the correlation times $\tau_{\mu \nu}$ in the equilibrium state. The correlation functions $\left\langle\Delta X_{\mu}(t) \Delta X_{v}(0)\right\rangle_{\mathrm{eq}}$ in the equilibrium state read $\left\langle\Delta X_{w}(t) \Delta X_{w}(0)\right\rangle_{\mathrm{eq}}=$ $4^{-1} C_{q^{2}}(t), \quad\left\langle\Delta X_{w}(t) \Delta X_{u}(0)\right\rangle_{\mathrm{eq}}=\left\langle\Delta X_{u}(t) \Delta X_{w}(0)\right\rangle_{\mathrm{eq}}=$ $-\left(\lambda_{w} / 4 T\right) C_{q^{2}}(t)$, and $\left\langle\Delta X_{u}(t) \Delta X_{u}(0)\right\rangle_{\mathrm{eq}}=\left(\lambda_{w} / 2 T\right)^{2} C_{q^{2}}(t)$ with $\quad C_{q^{2}}(t) \equiv\left\langle q^{2}(t) q^{2}(0)\right\rangle_{\mathrm{eq}}-\left\langle q^{2}\right\rangle_{\mathrm{eq}}^{2}$. Since the time-dependent part of these correlation functions $\left\langle\Delta X_{\mu}(t) \Delta X_{v}(0)\right\rangle_{\mathrm{eq}}$ is given by $C_{q^{2}}(t)$, all the correlation times should be equal: $\tau_{w w}=\tau_{u u}=\tau_{w u}=\tau_{u w}$. Therefore, they satisfy $\tau_{w w} \tau_{u u}=\tau_{w u}^{2}$, so that $g_{\mu \nu}^{(2)}$ is positive definite and $g_{\mu \nu}^{(i)}(i=1,2)$ can indeed be regarded as metric tensors. Using the nonstationary solution $p\left(q, t \mid q^{\prime}, t^{\prime}\right)$ (with $t \geqslant t^{\prime}$ ) of the Fokker-Planck equation (18) for constant $\lambda_{w}$ and $D=\beta^{-1}$ at their instantaneous values [80] and the stationary solution $p_{s}=p^{\text {eq }}$ obtained for $t-t^{\prime} \gg \tau_{\mu \nu}$, we get $\left\langle q^{2}(t) q^{2}(0)\right\rangle_{\mathrm{eq}}=\iint d q_{1} d q_{2} q_{1}^{2} q_{2}^{2} p\left(q_{1}, t \mid q_{2}, 0\right) p_{s}\left(q_{2}\right)=$ $\left(D / \lambda_{w}\right)^{2}\left(1+2 e^{-2 \lambda_{w} t / \gamma}\right)$ and $\left\langle q^{2}\right\rangle_{\mathrm{eq}}=\int d q q^{2} p_{s}(q)=D / \lambda_{w}$, and thus $C_{q^{2}}(t)=2\left(D / \lambda_{w}\right)^{2} e^{-2 \lambda_{w} t / \gamma}$. Therefore, we finally obtain

$$
\tau_{w w}=\tau_{u u}=\tau_{w u}=\frac{\gamma}{2 \lambda_{w}} .
$$

From $\sigma_{\mu \nu}$ evaluated for the instantaneous equilibrium state and Eq. (20), the metric tensor $g_{\mu \nu}^{(2)}$ reads

$$
g_{\mu \nu}^{(2)}=\frac{\gamma}{2 \lambda_{w}}\left[\begin{array}{cc}
\left(T / \lambda_{w}\right)^{2} & -T / \lambda_{w} \\
-T / \lambda_{w} & 1
\end{array}\right] .
$$

Remarkably, this metric is singular with a zero eigenvalue whose corresponding eigenvector (normalized to unity) is $\left[\left(\lambda_{w} / T\right)^{2}+1\right]^{-1 / 2}\left(\lambda_{w} / T, 1\right)^{\top}$. Thus, the direction of the zero eigenvalue of $g_{\mu \nu}^{(2)}$ is $d T / d \lambda_{w}=T / \lambda_{w}$, which is along a straight line on the $T-\lambda_{w}$ plane connecting each point at $\left(\lambda_{w}, T\right)$ and the origin [see Fig. 1(a)]. Since $g_{\mu \nu}^{(1)} \propto g_{\mu \nu}^{(2)}, g_{\mu \nu}^{(1)}$ also has a zero eigenvalue and the above argument applies to $g_{\mu \nu}^{(1)}$ as well. The other eigenvalue of $g_{\mu \nu}^{(2)}$ is $\gamma\left(2 \lambda_{w}\right)^{-1}[1+$ 
$\left.\left(T / \lambda_{w}\right)^{2}\right]$ and the corresponding eigenvector is orthogonal to the one for the zero eigenvalue, i.e., $\propto\left(-1, \lambda_{w} / T\right)^{\top}$.

It is noted that, along the path of the zero eigenvalue of $g_{\mu v}^{(i)}$ (i.e., a straight line on the $T-\lambda_{w}$ plane for a given value of $\left.T / \lambda_{w}\right)$, the mean value $\langle S\rangle_{\text {eq }}$ of the stochastic entropy is constant:

$$
\langle S\rangle_{\mathrm{eq}}=-\left\langle\ln p^{\mathrm{eq}}\right\rangle_{\mathrm{eq}}=2^{-1}\left[1+\ln 2 \pi-\ln \left(\lambda_{w} / T\right)\right] .
$$

Therefore, the isentropic process defined as the one that conserves $\langle S\rangle_{\text {eq }}[5,30]$ is along the path of the zero eigenvalue of $g_{\mu \nu}^{(i)}$.

Now we consider what is called the Brownian Carnot cycle (hereafter, we refer to it as the Carnot cycle for simplicity) realized in an experiment by Martínez et al. [5], which consists of two isothermal strokes at hot $\left(T_{h}\right)$ and cold $\left(T_{c}\right)$ temperatures, and two isentropic strokes [83] as shown in Fig. 1(b). The duration of the hot $(0 \rightarrow 1)$ and cold $(2 \rightarrow 3)$ isothermal strokes is denoted by $\tau_{h}$ and $\tau_{c}$, and that of the isentropic strokes $1 \rightarrow 2$ and $3 \rightarrow 4$ is denoted by $\tau_{1 \rightarrow 2}$ and $\tau_{3 \rightarrow 4}$, respectively. Thus the period $\tau$ of the cycle is given by $\tau=\tau_{\text {isoth }}+\tau_{\text {isen }}$ with $\tau_{\text {isoth }} \equiv \tau_{h}+\tau_{c}$ and $\tau_{\text {isen }} \equiv \tau_{1 \rightarrow 2}+$ $\tau_{3 \rightarrow 4}$ being the total duration of the isothermal strokes and isentropic strokes in a cycle, respectively.

Suppose the duration of each stroke in the cycle is given and it is large enough so that the linear approximation is valid. Now, we shall optimize the protocol of each stroke under its given duration to minimize $\langle A\rangle$ and $\overline{\left\langle\Delta A^{2}\right\rangle}$. First of all, it is noted that, since the isentropic strokes are along the path of the zero eigenvalue of $g_{\mu \nu}^{(1)}$ and $g_{\mu \nu}^{(2)}$, these strokes have no contribution to $\langle A\rangle, \overline{\left\langle\Delta A^{2}\right\rangle}$, and the thermodynamic lengths $\mathcal{L}^{(i)} \equiv \oint \sqrt{g_{\mu \nu}^{(i)} d \lambda_{\mu} d \lambda_{\nu}}$ of the cycle [84]. Therefore, the isentropic strokes are completely irrelevant to this optimization, and what we have to do is to optimize only the isothermal strokes to fulfill the lower bounds of the inequalities:

$$
\langle A\rangle=\left\langle A_{h}\right\rangle+\left\langle A_{c}\right\rangle \geqslant \frac{\left(\mathcal{L}_{h}^{(1)}\right)^{2}}{\tau_{h}}+\frac{\left(\mathcal{L}_{c}^{(1)}\right)^{2}}{\tau_{c}}
$$

$[27,85]$ and

$$
\overline{\left\langle\Delta A^{2}\right\rangle}=\overline{\left\langle\Delta A_{h}^{2}\right\rangle}+\overline{\left\langle\Delta A_{c}^{2}\right\rangle} \geqslant \frac{\left(\mathcal{L}_{h}^{(2)}\right)^{2}}{\tau_{h}}+\frac{\left(\mathcal{L}_{c}^{(2)}\right)^{2}}{\tau_{c}},
$$

where $A_{h}$ and $A_{c}$ are the dissipated availability in the hot and cold isothermal strokes, and $\mathcal{L}_{h}^{(i)} \equiv \int_{0 \rightarrow 1} \sqrt{g_{\mu \nu}^{(i)} d \lambda_{\mu} d \lambda_{\nu}}$ and $\mathcal{L}_{c}^{(i)} \equiv \int_{2 \rightarrow 3} \sqrt{g_{\mu \nu}^{(i)} d \lambda_{\mu} d \lambda_{\nu}}$ are the thermodynamic length of the path of the hot and cold isothermal strokes, respectively. Writing $\lambda_{w, i}$ for $\lambda_{w}$ at node $i$, from Eqs. (21) and (16) we obtain $\mathcal{L}_{h}^{(i)}=\sqrt{2^{i-1} \gamma T_{h}^{i}}\left(\lambda_{w, 1}^{-1 / 2}-\lambda_{w, 0}^{-1 / 2}\right)(i=1,2)$. Since $T_{h} / \lambda_{w, 1}=T_{c} / \lambda_{w, 2}$ and $T_{h} / \lambda_{w, 0}=T_{c} / \lambda_{w, 3}$, we further get $\mathcal{L}_{c}^{(i)}=\left(T_{c} / T_{h}\right)^{(i-1) / 2} \mathcal{L}_{h}^{(i)}(i=1,2)$. As has been discussed for Eq. (10), the equalities of Eqs. (23) and (24) hold when $g_{\mu \nu}^{(i)}(t) \dot{\lambda}_{\mu}(t) \dot{\lambda}_{\nu}(t)$ is constant in time, or equivalently, when we sweep the parameter $\lambda_{\mu}$ in a way such that the time interval to traverse the path element $d \lambda_{\mu}$ is proportional to the thermodynamic length $d \mathcal{L}^{(i)}$ of this path element. Since $g_{\mu \nu}^{(i)} \dot{\lambda}_{\mu} \dot{\lambda}_{v}=g_{w w}^{(i)} \dot{\lambda}_{w}^{2}$ in the isothermal strokes, the above condition is satisfied by sweeping as

$$
\left|\dot{\lambda}_{w}\right| \propto \lambda_{w}^{3 / 2}
$$

and we get $\langle A\rangle=\left(\tau_{h}^{-1}+\tau_{c}^{-1}\right)\left(\mathcal{L}_{h}^{(1)}\right)^{2}$ and $\overline{\left\langle\Delta A^{2}\right\rangle}=\left[\tau_{h}^{-1}+\right.$ $\left.\left(T_{c} / T_{h}\right) \tau_{c}^{-1}\right]\left(\mathcal{L}_{h}^{(2)}\right)^{2}$. It is noted that, since $g_{\mu \nu}^{(1)}$ and $g_{\mu \nu}^{(2)}$ are related through Eq. (16) and $T$ is constant in each isothermal stroke, the optimal protocol (25) applies to both $\langle A\rangle$ and $\overline{\left\langle\Delta A^{2}\right\rangle}$. Namely, once the duration of each stroke is given, both $\langle A\rangle$ and $\overline{\left\langle\Delta A^{2}\right\rangle}$ for the Carnot cycle are optimized simultaneously, which is in contrast to the trade-off optimization between, e.g., work fluctuation and efficiency [74].

As can be seen from the above discussion, such simultaneous minimization of $\langle A\rangle$ and $\overline{\left\langle\Delta A^{2}\right\rangle}$ by the same protocol within finite time is possible only for cycles solely consisting of isothermal strokes and isentropic strokes. First of all, isentropic strokes have zero contribution to $\langle A\rangle$ and $\overline{\left\langle\Delta A^{2}\right\rangle}$ because of the zero eigenvalue of the metric in our system. In addition, only isothermal strokes are nontrivial strokes with nonzero $\langle A\rangle$ and $\overline{\left\langle\Delta A^{2}\right\rangle}$ that allow us to minimize both $\langle A\rangle$ and $\overline{\left\langle\Delta A^{2}\right\rangle}$ simultaneously by the same protocol $\lambda_{\mu}(t)$. Among cycles whose number of strokes $N_{s}$ is less than or equal to 5 , the Carnot cycle is the only possibility to form a meaningful cycle with nonzero work output solely with isothermal and isentropic strokes [86]. Therefore, when the duration of each stroke is given, the Carnot cycle is the only case with $N_{s} \leqslant 5$ which allows us to simultaneously minimize $\langle A\rangle$ and $\overline{\left\langle\Delta A^{2}\right\rangle}$.

It is noted that the existence of the zero eigenvalue is essential for the above simultaneous minimization, and such a singular metric is due to the scale invariance of the equilibrium state. In the overdamped case, once the trapping potential is scale invariant, i.e., $V_{\lambda_{w}}$ satisfies $V_{\lambda_{w}}(a q)=|a|^{\alpha} V_{\lambda_{w}}(q)$ for some $\alpha$ with $a$ being the scaling factor, so is the system itself, and as a consequence, the metric becomes singular. A power-law function is a representative example of the scale invariant function, and the above simultaneous minimization of $\langle A\rangle$ and $\overline{\left\langle\Delta A^{2}\right\rangle}$ is possible also for a general power-law trapping potential $V_{\lambda_{w}}(q) \propto \lambda_{w}|q|^{k}$ with positive $k$ [79].

If only the total duration of the isothermal strokes $\tau_{\text {isoth }}$ is given instead of $\tau_{h}$ and $\tau_{c}$ separately, we can further optimize to reduce either $\langle A\rangle$ or $\overline{\left\langle\Delta A^{2}\right\rangle}$, respectively. This optimization holds irrespective of the duration $\tau_{\text {isen }}$ and the protocol of the isentropic strokes, provided the driving in these strokes is slow enough. From the condition that $g_{\mu \nu}^{(i)}(t) \dot{\lambda}_{\mu}(t) \dot{\lambda}_{v}(t)=g_{w w}^{(i)} \dot{\lambda}_{w}^{2}$ is constant at the same value for both the two isothermal strokes, the optimal protocol is $\left|\dot{\lambda}_{w}\right| \propto \lambda_{w}^{3 / 2} T^{-i+1}(i=1,2)$, which yields $\tau_{h}=\tau_{c}=\tau_{\text {isoth }} / 2$ to optimize $\langle A\rangle$ and $\tau_{h} / \sqrt{T_{h}}=$ $\tau_{c} / \sqrt{T_{c}}$ [i.e., $\tau_{h}=\tau_{\text {isoth }} /\left(1+\sqrt{T_{c} / T_{h}}\right)$ and $\tau_{c}=\tau_{\text {isoth }}-\tau_{h}$ ] to optimize $\overline{\left\langle\Delta A^{2}\right\rangle}$ with their minimum vales $\langle A\rangle=\left(\mathcal{L}^{(1)}\right)^{2} / \tau_{\text {isoth }}$ and $\overline{\langle\Delta A\rangle^{2}}=\left(\mathcal{L}^{(2)}\right)^{2} / \tau_{\text {isoth }}$, respectively.

Finally, we discuss how much the optimized protocol given by Eq. (25) improves the dissipated availability and its fluctuation of the Carnot cycle compared to the one in the current experiment [5]. In this experiment, $\lambda_{w}(t)$ in Eq. (17) is controlled by the following protocol: $\lambda_{w}(t)=$ $f(t)$ for $0 \leqslant t \leqslant \tau / 2$ and $\lambda_{w}(t)=f(\tau-t)$ for $\tau / 2 \leqslant t \leqslant \tau$ with $f(t)=4\left(\lambda_{w, 2}-\lambda_{w, 0}\right)(t / \tau)^{2}+\lambda_{w, 0}$. The experimental parameter values are as follows: $T_{c}=300 \mathrm{~K}, T_{h}=525 \mathrm{~K}$,

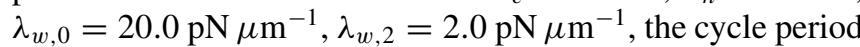
$\tau=200 \mathrm{~ms}$, and the duration of each stroke is $\tau_{0 \rightarrow 1}=0.26 \tau$, $\tau_{1 \rightarrow 2}=0.24 \tau, \tau_{2 \rightarrow 3}=0.25 \tau$, and $\tau_{3 \rightarrow 4}=0.25 \tau$. In addition, the friction coefficient is estimated as $\gamma=8.4{\mathrm{pN} \mu \mathrm{m}^{-1} \mathrm{~ms}}^{-}$ 
[79]. From these parameter values, the ratio of the dissipated availabilities for the experimental protocol and the optimized protocol given by Eq. (25) and that for their fluctuations are calculated to be [79]

$$
\frac{\left\langle A_{\text {opt }}\right\rangle}{\left\langle A_{\text {expt }}\right\rangle}=0.65, \quad \frac{\overline{\left\langle\Delta A_{\text {opt }}^{2}\right\rangle}}{\overline{\left\langle\Delta A_{\text {expt }}^{2}\right\rangle}}=0.70 .
$$

Here, the subscripts "opt" and "expt" represent quantities obtained by the optimized and the experimental protocols, respectively. The optimized protocol improves the dissipated availability by $35 \%$ and its fluctuation by $30 \%$. This means that the above optimization improves not only the efficiency $\epsilon$, which is given by $\epsilon \equiv\langle W\rangle /\langle U\rangle \simeq 1-\langle A\rangle / \mathcal{W}$ with $\mathcal{W}(=$ $\langle\mathcal{W}\rangle$ ) being the work output by the quasistatic cycle [27], but also the stability of the fluctuating efficiency $\mathcal{E} \equiv W / U \simeq$ $1-A / \mathcal{W}$ characterized by $\overline{\left\langle\Delta \mathcal{E}^{2}\right\rangle} \simeq \overline{\left\langle\Delta A^{2}\right\rangle} / \mathcal{W}^{2}$. Since the fluctuation of the stochastic efficiency $\mathcal{E}$ is non-negligible in the Brownian Carnot engine (in the experiment of [5], the variance of $\mathcal{E}$ is of order unity, and the distribution function of $\mathcal{E}$ spreads even to the negative side), it is crucially important to reduce $\overline{\left\langle\Delta A^{2}\right\rangle}$ as well as $\langle A\rangle$.

Concluding remarks. In summary, we have formulated finite-time thermodynamics of fluctuations in microscopic heat engines, which universally holds for the slow-driving regime and the coarse-grained timescale. This formalism provides a geometric description of the fluctuation of the dissipation whose metric is found to be consistent with the relation analogous to the fluctuation-dissipation relation. Applying our framework to the Carnot cycle, it has been found that both the average and the fluctuation of the dissipation can be minimized simultaneously when the duration of each stroke is given. Interestingly, we have seen that the scale invariance of the equilibrium state is essential for this simultaneous minimization. The benefit of this optimized protocol has been demonstrated for the current experiment [5].

Our framework should find broad applications to design not only energy efficient but also stable microscopic thermal machines. While we have considered classical systems, developing a corresponding formalism for quantum systems constitutes an important and challenging future problem. Further analysis based on Riemannian geometry for systems with a nonsingular metric, such as a classical or quantum two-level system, etc., would lead to a deeper understanding on thermodynamics of fluctuations. For example, geometric interpretation of thermodynamics of fluctuations provided in our work may lead to another type of universal relations and bounds on fluctuations of the performance of microscopic heat engines distinct from thermodynamic uncertainty relations [87-91].

Acknowledgments. This work was supported by NSF of China (Grants No. 11975199 and No. 11674283), by the Zhejiang Provincial Natural Science Foundation Key Project (Grant No. LZ19A050001), and by the Zhejiang University 100 Plan.
[1] T. Hugel, N. B. Holland, A. Cattani, L. Moroder, M. Seitz, and H. E. Gaub, Single-Molecule Optomechanical Cycle, Science 296, 1103 (2002).

[2] P. G. Steeneken, K. Le Phan, M. J. Goossens, G. E. J. Koops, G. J. A. M. Brom, C. van der Avoort, and J. T. M. van Beek, Piezoresistive heat engine and refrigerator, Nat. Phys. 7, 354 (2011).

[3] V. Blickle and C. Bechinger, Realization of a micrometre-sized stochastic heat engine, Nat. Phys. 8, 143 (2012).

[4] P. A. Quinto-Su, A microscopic steam engine implemented in an optical tweezer, Nat. Commun. 5, 5889 (2014).

[5] I. A. Martínez, É. Roldán, L. Dinis, D. Petrov, J. M. R. Parrondo, and R. A. Rica, Brownian Carnot engine, Nat. Phys. 12, 67 (2016).

[6] M. Serra-Garcia, A. Foehr, M. Moleron, J. Lydon, C. Chong, and C. Daraio, Mechanical Autonomous Stochastic Heat Engine, Phys. Rev. Lett. 117, 010602 (2016).

[7] I. A. Martínez, É. Roldán, L. Dinis, and R. A. Rica, Colloidal heat engines: a review, Soft Matter 13, 22 (2017).

[8] J. Klaers, S. Faelt, A. Imamoglu, and E. Togan, Squeezed Thermal Reservoirs as a Resource for a Nanomechanical Engine beyond the Carnot Limit, Phys. Rev. X 7, 031044 (2017).

[9] A. Argun, J. Soni, L. Dabelow, S. Bo, G. Pesce, R. Eichhorn, and G. Volpe, Experimental realization of a minimal microscopic heat engine, Phys. Rev. E 96, 052106 (2017).

[10] K. Sekimoto, Langevin Equation and Thermodynamics, Prog. Theor. Phys. Suppl. 130, 17 (1998).
[11] K. Sekimoto, Stochastic Energetics, Lecture Notes in Physics Vol. 799 (Springer, Berlin, 2010).

[12] C. Jarzynski, Equalities and Inequalities: Irreversibility and the Second Law of Thermodynamics at the Nanoscale, Annu. Rev. Condens. Matter Phys. 2, 329 (2011).

[13] U. Seifert, Stochastic thermodynamics, fluctuation theorems and molecular machines, Rep. Prog. Phys. 75, 126001 (2012).

[14] C. Van den Broeck and M. Esposito, Ensemble and trajectory thermodynamics: A brief introduction, Phys. A (Amsterdam, Neth.) 418, 6 (2015).

[15] S. Ciliberto, Experiments in Stochastic Thermodynamics: Short History and Perspectives, Phys. Rev. X 7, 021051 (2017).

[16] G. Nicolis and Y. De Decker, Stochastic Thermodynamics of Brownian Motion, Entropy 19, 434 (2017).

[17] C. Bustamante, J. Liphardt, and F. Ritort, The Nonequilibrium Thermodynamics of Small Systems, Phys. Today 58(7), 43 (2005).

[18] S. Krishnamurthy, S. Ghosh, D. Chatterji, R. Ganapathy, and A. K. Sood, A micrometre-sized heat engine operating between bacterial reservoirs, Nat. Phys. 12, 1134 (2016).

[19] P. Pietzonka, É. Fodor, C. Lohrmann, M. E. Cates, and U. Seifert, Autonomous Engines Driven by Active Matter: Energetics and Design Principles, Phys. Rev. X 9, 041032 (2019).

[20] É. Fodor and M. E. Cates, Active engines: Thermodynamics moves forward, Europhys. Lett. 134, 10003 (2021).

[21] S. Toyabe and M. Sato, Nonequilibrium Fluctuations in Biological Strands, Machines, and Cells, J. Phys. Soc. Jpn. 84, 102001 (2015). 
[22] K. Sekimoto, F. Takagi, and T. Hondou, Carnot's cycle for small systems: Irreversibility and cost of operations, Phys. Rev. E 62, 7759 (2000)

[23] T. Schmiedl and U. Seifert, Efficiency at maximum power: An analytically solvable model for stochastic heat engines, Europhys. Lett. 81, 200003 (2008).

[24] A. Dechant, N. Kiesel, and E. Lutz, All-Optical Nanomechanical Heat Engine, Phys. Rev. Lett. 114, 183602 (2015).

[25] K. Brandner, K. Saito, and U. Seifert, Thermodynamics of Micro- and Nano-Systems Driven by Periodic Temperature Variations, Phys. Rev. X 5, 031019 (2015).

[26] A. Dechant, N. Kiesel, and E. Lutz, Underdamped stochastic heat engine at maximum efficiency, Europhys. Lett. 119, 50003 (2017).

[27] K. Brandner and K. Saito, Thermodynamic Geometry of Microscopic Heat Engines, Phys. Rev. Lett. 124, 040602 (2020).

[28] P. Strasberg, C. W. Wächtler, and G. Schaller, Autonomous Implementation of Thermodynamic Cycles at the Nanoscale, Phys. Rev. Lett. 126, 180605 (2021).

[29] P. Jop, A. Petrosyan, and S. Ciliberto, Work and dissipation fluctuations near the stochastic resonance of a colloidal particle, Europhys. Lett. 81, 50005 (2008).

[30] I. A. Martínez, É. Roldán, L. Dinis, D. Petrov, and R. A. Rica, Adiabatic Processes Realized with a Trapped Brownian Particle, Phys. Rev. Lett. 114, 120601 (2015).

[31] J. Hoppenau, M. Niemann, and A. Engel, Carnot process with a single particle, Phys. Rev. E 87, 062127 (2013).

[32] V. Holubec, An exactly solvable model of a stochastic heat engine: optimization of power, power fluctuations and efficiency, J. Stat. Mech. (2014) P05022.

[33] S. Rana, P. S. Pal, A. Saha, and A. M. Jayannavar, Singleparticle stochastic heat engine, Phys. Rev. E 90, 042146 (2014).

[34] L. Cerino, A. Puglisi, and A. Vulpiani, Kinetic model for the finite-time thermodynamics of small heat engines, Phys. Rev. E 91, 032128 (2015).

[35] V. Holubec and A. Ryabov, Work and power fluctuations in a critical heat engine, Phys. Rev. E 96, 030102(R) (2017).

[36] V. Holubec and A. Ryabov, Cycling Tames Power Fluctuations near Optimum Efficiency, Phys. Rev. Lett. 121, 120601 (2018).

[37] A. Dechant, Multidimensional thermodynamic uncertainty relations, J. Phys. A: Math. Theor. 52, 035001 (2019).

[38] K. Ito, C. Jiang, and G. Watanabe, Universal Bounds for Fluctuations in Small Heat Engines, arXiv:1910.08096.

[39] S. Saryal, M. Gerry, I. Khait, D. Segal, and B. K. Agarwalla, Universal Bounds on Fluctuations in Continuous Thermal Machines, Phys. Rev. Lett. 127, 190603 (2021).

[40] S. Mohanta, S. Saryal, and B. K. Agarwalla, Universal bounds on cooling power and cooling efficiency for autonomous absorption refrigerators, arXiv:2106.12809.

[41] V. Holubec and A. Ryabov, Fluctuations in heat engines, J. Phys. A: Math. Theor. 55, 013001 (2021).

[42] G. Verley, M. Esposito, T. Willaert, and C. Van den Broeck, The unlikely Carnot efficiency, Nat. Commun. 5, 4721 (2014).

[43] G. Verley, T. Willaert, C. Van den Broeck, and M. Esposito, Universal theory of efficiency fluctuations, Phys. Rev. E 90, 052145 (2014).

[44] K. Proesmans and C. Van den Broeck, Stochastic efficiency: five case studies, New. J. Phys. 17, 065004 (2015).
[45] K. Proesmans, C. Driesen, B. Cleuren, and C. Van den Broeck, Efficiency of single-particle engines, Phys. Rev. E 92, 032105 (2015).

[46] J.-M. Park, H.-M. Chun, and J. D. Noh, Efficiency at maximum power and efficiency fluctuations in a linear Brownian heatengine model, Phys. Rev. E 94, 012127 (2016).

[47] A. Saha, R. Marathe, P. S. Pal, and A. M. Jayannavar, Stochastic heat engine powered by active dissipation, J. Stat. Mech. (2018) 113203.

[48] S. K. Manikandan, L. Dabelow, R. Eichhorn, and S Krishnamurthy, Efficiency Fluctuations in Microscopic Machines, Phys. Rev. Lett. 122, 140601 (2019).

[49] H. Vroylandt, M. Esposito, and G. Verley, Efficiency Fluctuations of Stochastic Machines Undergoing a Phase Transition, Phys. Rev. Lett. 124, 250603 (2020).

[50] N. A. Sinitsyn, Fluctuation relation for heat engines, J. Phys. A 44, 405001 (2011)

[51] P. S. Pal, S. Lahiri, and A. M. Jayannavar, Transient exchange fluctuation theorems for heat using a Hamiltonian framework: Classical and quantum regimes, Phys. Rev. E 95, 042124 (2017).

[52] K. Proesmans and C. Van den Broeck, Discrete-time thermodynamic uncertainty relation, Europhys. Lett. 119, 20001 (2017).

[53] P. Pietzonka and U. Seifert, Universal Trade-Off between Power, Efficiency, and Constancy in Steady-State Heat Engines, Phys. Rev. Lett. 120, 190602 (2018).

[54] A. C. Barato, R. Chetrite, A. Faggionato, and D. Gabrielli, Bounds on current fluctuations in periodically driven systems, New J. Phys. 20, 103023 (2018).

[55] T. Koyuk, U. Seifert, and P. Pietzonka, A generalization of the thermodynamic uncertainty relation to periodically driven systems, J. Phys. A: Math. Theor. 52, 02LT02 (2019).

[56] T. Koyuk and U. Seifert, Operationally Accessible Bounds on Fluctuations and Entropy Production in Periodically Driven Systems, Phys. Rev. Lett. 122, 230601 (2019).

[57] A. M. Timpanaro, G. Guarnieri, J. Goold, and G. T. Landi, Thermodynamic Uncertainty Relations from Exchange Fluctuation Theorems, Phys. Rev. Lett. 123, 090604 (2019)2019.

[58] T. Kamijima, S. Otsubo, Y. Ashida, and T. Sagawa, Higherorder efficiency bound and its application to nonlinear nanothermoelectrics, Phys. Rev. E 104, 044115 (2021).

[59] G.-H. Xu and G. Watanabe, Correlation-enhanced Stability of Microscopic Cyclic Heat Engines, arXiv:2111.09508.

[60] F. Weinhold, Metric geometry of equilibrium thermodynamics, J. Chem. Phys. 63, 2479 (1975).

[61] G. Ruppeiner, Thermodynamics: A Riemannian geometric model, Phys. Rev. A 20, 1608 (1979).

[62] P. Salamon, J. Nulton, and E. Ihrig, On the relation between entropy and energy versions of thermodynamic length, J. Chem. Phys. 80, 436 (1984).

[63] R. Gilmore, Length and curvature in the geometry of thermodynamics, Phys. Rev. A 30, 1994 (1984).

[64] F. Schlögl, Thermodynamic metric and stochastic measures, Z. Phys. B 59, 449 (1985).

[65] D. Brody and N. Rivier, Geometrical aspects of statistical mechanics, Phys. Rev. E 51, 1006 (1995).

[66] G. Ruppeiner, Riemannian geometry in thermodynamic fluctuation theory, Rev. Mod. Phys. 67, 605 (1995). 
[67] P. Salamon and R. S. Berry, Thermodynamic Length and Dissipated Availability, Phys. Rev. Lett. 51, 1127 (1983).

[68] B. Andresen, P. Salamon, and R. S. Berry, Thermodynamics in finite time, Phys. Today 37(9), 62 (1984).

[69] J. Nulton, P. Salamon, B. Andresen, and Q. Anmin, Quasistatic processes as step equilibrations, J. Chem. Phys. 83, 334 (1985).

[70] G. E. Crooks, Measuring Thermodynamic Length, Phys. Rev. Lett. 99, 100602 (2007).

[71] D. A. Sivak and G. E. Crooks, Thermodynamic Metrics and Optimal Paths, Phys. Rev. Lett. 108, 190602 (2012).

[72] P. R. Zulkowski, D. A. Sivak, G. E. Crooks, and M. R. DeWeese, Geometry of thermodynamic control, Phys. Rev. E 86, 041148 (2012).

[73] T. Van Vu and Y. Hasegawa, Geometrical Bounds of the Irreversibility in Markovian Systems, Phys. Rev. Lett. 126, 010601 (2021).

[74] H. J. D. Miller and M. Mehboudi, Geometry of Work Fluctuations versus Efficiency in Microscopic Thermal Machines, Phys. Rev. Lett. 125, 260602 (2020).

[75] A. G. Frim and M. R. DeWeese, Optimal finite-time Brownian Carnot engine, arXiv:2107.05673.

[76] C. A. Plata, D. Guéry-Odelin, E. Trizac, and A. Prados, Optimal work in a harmonic trap with bounded stiffness, Phys. Rev. E 99, 012140 (2019).

[77] C. A. Plata, D. Guéry-Odelin, E. Trizac, and A. Prados, Building an irreversible Carnot-like heat engine with an overdamped harmonic oscillator, J. Stat. Mech. (2020) 093207.

[78] C. A. Plata, D. Guéry-Odelin, E. Trizac, and A. Prados, Finitetime adiabatic processes: Derivation and speed limit, Phys. Rev. E 101, 032129 (2020).

[79] See Supplemental Material at http://link.aps.org/supplemental/ 10.1103/PhysRevResearch.4.L012008 for details, which includes Refs. [5,27,75,80,81].

[80] H. Risken, The Fokker-Planck Equation, 2nd ed. (Springer, Berlin, 1988).
[81] P. Mestres, I. A. Martinez, A. Ortiz-Ambriz, R. A. Rica, and E. Roldan, Realization of nonequilibrium thermodynamic processes using external colored noise, Phys. Rev. E 90, 032116 (2014).

[82] C. Gardiner, Stochastic Methods-A Handbook for the Natural and Social Sciences, 4th ed. (Springer, Berlin, 2009).

[83] In the experiment of Ref. [5], adiabatic strokes in the conventional Carnot cycle are replaced by isentropic strokes with a constant Shannon entropy of the working substance. In the present overdamped case, such an isentropic process is given by $T / \lambda_{w}=$ const instead of $T^{2} / \lambda_{w}=$ const for the underdamped case.

[84] It is noted that, although a separate isentropic stroke gives nonzero fluctuation of $A$ due to the contribution from its end points, the isentropic strokes in a cycle make no contribution to $\overline{\left\langle\Delta A^{2}\right\rangle}$.

[85] K. Sekimoto and S.-I. Sasa, Complementarity Relation for Irreversible Process Derived from Stochastic Energetics, J. Phys. Soc. Jpn. 66, 3326 (1997).

[86] Here we exclude the cycles with trivial strokes in which the system moves along the same path in the parameter space back and forth.

[87] A. C. Barato and U. Seifert, Thermodynamic Uncertainty Relation for Biomolecular Processes, Phys. Rev. Lett. 114, 158101 (2015).

[88] T. R. Gingrich, J. M. Horowitz, N. Perunov, and J. L. England, Dissipation Bounds All Steady-State Current Fluctuations, Phys. Rev. Lett. 116, 120601 (2016).

[89] K. Macieszczak, K. Brandner, and J. P. Garrahan, Unified Thermodynamic Uncertainty Relations in Linear Response, Phys. Rev. Lett. 121, 130601 (2018).

[90] U. Seifert, From Stochastic Thermodynamics to Thermodynamic Inference, Annu. Rev. Condens. Matter Phys. 10, 171 (2019).

[91] G. Falasco, M. Esposito, and J.-C. Delvenne, Unifying thermodynamic uncertainty relations, New J. Phys. 22, 053046 (2020). 